\title{
Anti-metastatic effects of Aidi on human esophageal squamous cell carcinoma by inhibiting epithelial-mesenchymal transition and angiogenesis
}

\author{
QINGTONG SHI $^{*}$, YALI DIAO ${ }^{1 *}$, FENG JIN $^{2}$ and ZHIYAN DING ${ }^{3}$ \\ ${ }^{1}$ Department of Thoracic Surgery, The Affiliated Hospital of Yangzhou University; \\ ${ }^{2}$ Institute of Combining Traditional Chinese and Western Medicine, Medical College; ${ }^{3}$ Department of Pathology, \\ The Affiliated Hospital of Yangzhou University, Yangzhou University, Yangzhou, Jiangsu 225009, P.R. China
}

Received November 29, 2017; Accepted April 5, 2018

DOI: $10.3892 / \mathrm{mmr} .2018 .8976$

\begin{abstract}
Aidi injection, a proprietary Chinese medicine, has been widely used for the treatment of cancer. However, its effects and potential mechanism in esophageal squamous cell carcinoma (ESCC) have not yet been characterized. The aim of the present study was to identify the mechanism underlying the anti-metastatic effects of treatment with Aidi. To test the effects and mechanism, EC9706 and KYSE70 cells were selected for in vitro experiments. In vivo, the anti-metastatic effects of Aidi injection on a nude mouse peritoneal metastasis model were examined, and the mechanisms were assessed with immunohistochemical staining. A cell proliferation assay demonstrated that treatment with more than $3 \mathrm{mg} / \mathrm{ml}$ Aidi for 24 or $48 \mathrm{~h}$ significantly inhibited the proliferation of EC9706 $(\mathrm{P}<0.01)$ and KYSE70 cells $(\mathrm{P}<0.05$, $\mathrm{P}<0.01)$. Subsequent experiments assessed cell migration, invasion and vasculogenic mimicry (VM) formation, with 5 -fluorouracil serving as a positive control. It was observed that treatment with Aidi inhibited cell migration, invasion and VM formation. Furthermore, it was identified that treatment with Aidi inhibited epithelial-mesenchymal transition (EMT) signaling and the expression of vascular endothelial growth
\end{abstract}

Correspondence to: Dr Zhiyan Ding, Department of Pathology, The Affiliated Hospital of Yangzhou University, Yangzhou University, 368 Hanjiang Middle Road, Yangzhou, Jiangsu 225009, P.R. China

E-mail: dingzy39@163.com

*Contributed equally

Abbreviations: DMEM, Dulbecco's modified Eagle's medium; EMT, epithelial-mesenchymal transition; ESCC, esophageal squamous cell carcinoma; FBS, fetal bovine serum; HUVECs, human umbilical vein endothelial cells; TWIST1, Twist-related protein 1; VEGF-A, vascular endothelial growth factor-A; VM, vasculogenic mimicry; ZEB1, zinc finger E-box-binding homeobox 1

Key words: Aidi injection, metastasis, angiogenesis, EMT, ESCC factor A (VEGF-A) in EC9706 and KYSE70 cells, using western blotting. In the in vivo experiments, Aidi injection effectively suppressed tumor metastasis in the mouse tumor model. Additionally, the expression of vimentin and vascular endothelial growth factor was decreased, and the expression of cadherin-1 was increased in the tumor tissue. The present results suggested that treatment with Aidi may inhibit tumor metastasis in ESCC through the inhibition of EMT signaling and angiogenesis.

\section{Introduction}

Esophageal cancer is one of the most common types of cancer worldwide, and is associated with a particularly poor prognosis. Esophageal squamous cell carcinoma (ESCC) is the most common pathological type of esophageal cancer, accounting for $70 \%$ of cases (1). The incidence and mortality rates for ESCC are particularly high in Asian countries compared with Western countries (2). A combination of treatments, including surgery and chemotherapy, is the primary method for treating ESCC $(3,4)$. However, the prognosis of esophageal cancer remains poor, and the overall five-year survival rate was reported to be $15-25 \%$ (5). Tumor metastasis is the main cause of mortality associated with esophageal squamous cell carcinoma (6). Due to the lack of serous layer in the esophageal wall, direct infiltration and metastasis can occur in the early stage of esophageal cancer (7). It has been reported that 56\% of patients have suffered from blood vessel metastasis and 32\% of patients have suffered from lymph metastasis during tumor invasion of the submucosal tissue (8). The early symptoms and signs of esophageal cancer are not easily distinguished; however, when identified, the disease tends to be within the middle and late stage of pathogenesis. Subsequently, distant metastasis occurs, leading to poor prognosis (4). Therefore, the mechanism of the invasion and metastasis of esophageal cancer has been a focus of research.

Aidi, an extract of ginseng, Astragalus membranaceus, Acanthopanax and Mylabris, has been researched and manufactured in abundance for clinical use in China since 2002 (9). Aidi injection has been widely used for the treatment of a range of cancer types, including ESCC (10), gastric carcinoma (11), 
primary liver cancer (12) and colorectal cancer (13). Modern pharmacological research has demonstrated that treatment with Aidi produces various pharmacological effects, including anti-tumor and immune regulatory actions. The chemical constituents of Aidi have been identified through spectral data from chromatography on Sephadex LH-20 gel columns, and reverse phase semi-preparative high-performance liquid chromatography; a total of 22 compounds were isolated and identified (14). The results showed that the 6 compounds were from Astragalus membranaceus, 12 compounds were from ginseng, 4 compounds came from Acanthopanax. The evaluation of pharmacological and toxicological activity in compound preparation is still in progress. The anti-tumor effects of treatment with Aidi include the induction of apoptosis, the inhibition of cell proliferation and angiogenesis, and the relief of chemotherapy-associated side effects $(15,16)$. However, the therapeutic effects of Aidi on the inhibition of metastasis in ESCC are unclear.

The mechanisms of invasion and metastasis of tumor are very complex involves hypoxia microenvironment, angiogenesis, epithelial-mesenchymal transformation (EMT), and network regulation of numerous signaling pathways and so on (17). In this study, we would elucidate the anti-metastatic effects of treatment with Aidi in ESCC, and identify the possible underlying mechanisms angiogenesis and EMT in the anti-metastatic effects.

\section{Materials and methods}

Cell culture and drugs. The human ESCC cell lines EC9706 and KYSE70 (Cell Bank of Chinese Academy of Sciences, Shanghai Institute of Cell Biology, Shanghai, China) were cultured in RPMI-1640 medium supplemented with $10 \%$ fetal bovine serum (FBS; both Gibco; Thermo Fisher Scientific, Inc., Waltham, MA, USA) in $5 \% \mathrm{CO}_{2}$ at $37^{\circ} \mathrm{C}$. Human umbilical vein endothelial cells (HUVECs; Zhong Qiao Xin Zhou Biotech, Shanghai, China) were cultured in high-glucose Dulbecco's modified Eagle's medium (DMEM; Gibco; Thermo Fisher Scientific, Inc.) containing $20 \% \mathrm{FBS}$ in $5 \% \mathrm{CO}_{2}$ at $37^{\circ} \mathrm{C}$. Aidi was purchased from Guizhou Yibai Pharmaceutical Co., Ltd. (Guizhou, China) with an initial concentration of $0.3 \mathrm{~g} / \mathrm{ml}$. 5-Fluorouracil (5-Fu; $0.025 \mathrm{~g} / \mathrm{ml}$ ) was purchased from Jin Yao Amino Acid Co., Ltd. (Tianjin, China). The drugs were diluted to the required concentration in a sterile environment with the cell culture media mentioned above, and were used in in vitro experiments; drugs diluted with sterile saline were used in in vivo experiments.

Cell proliferation assay. The cells $\left(2 \times 10^{3}\right)$ were cultured in 96 -well plates for $24 \mathrm{~h}$ at $37^{\circ} \mathrm{C}$. Culture medium $(100 \mu \mathrm{l})$ containing $1.5,3,6,12,24,48$ or $96 \mathrm{mg} / \mathrm{ml}$ Aidi was added following removal of the original medium. The cells were cultured for 24 or $48 \mathrm{~h}$ at $37^{\circ} \mathrm{C}$. A volume of $15 \mu 15 \mathrm{mg} / \mathrm{ml}$ MTT (Sigma-Aldrich; Merck KGaA, Darmstadt, Germany) was added and incubated for $4 \mathrm{~h}$, and subsequently $180 \mu \mathrm{l}$ dimethyl sulfoxide (Amresco, LLC, Solon, OH, USA) was added. Once the bluish violet crystalline formazan had dissolved completely with gentle shaking for $10 \mathrm{~min}$, the absorbance (A) was detected at $490 \mathrm{~nm}$ using a microplate reader (Bio-Rad Laboratories, Inc., Hercules, CA,
USA). The cell growth rate (\%) was determined as follows: $\left(\mathrm{A}_{\text {control group }}-\mathrm{A}_{\text {observed group }}\right) / \mathrm{A}_{\text {control group }} \mathrm{x} 100$.

Cell morphology observation. A total of $1 \times 10^{5}$ cells per well were cultured in 6 -well plates for $24 \mathrm{~h}$ at $37^{\circ} \mathrm{C}$. Then cells were treated with 3,6 or $12 \mathrm{mg} / \mathrm{ml}$ Aidi, $32 \mathrm{mg} / 1$ 5-Fu, or without any drugs respectively for $24 \mathrm{~h}$ at $37^{\circ} \mathrm{C}$. Cells were observed and photographed using bright field microscopy (magnification, x100, BX41; Olympus Corporation, Tokyo Japan).

Wound-healing assay. To detect the rate of migration, a wound-healing assay was performed. Cells were cultured on 6-well plates for $24 \mathrm{~h}$, prior to being treated with 6,12 or $24 \mathrm{mg} / \mathrm{ml} \mathrm{Aidi,} 32 \mathrm{mg} / \mathrm{l}$ 5-Fu, or without drugs for $24 \mathrm{~h}$. A pipette tip was used to scratch the monolayer in each well. Detached cells were removed by washing twice with PBS. The remaining adherent cells were cultured with complete culture medium for $24 \mathrm{~h}$. The extent of space filling, representing the cell migration, was evaluated using bright field microscopy (magnification, x100, BX41; Olympus Corporation, Tokyo Japan). The migratory capacity of cells was calculated as (scratch width of the treatment group-scratch width of the control group)/scratch width of the control group x100.

Matrigel invasion assay. To detect the invasive ability of the cells, Corning Matrigel Invasion Chambers (Corning Incorporated, Corning, NY, USA) were used. Matrigel was diluted with RPMI-1640 medium at a ratio of 1:8 and used to coat the upper chamber. RPMI-1640 or DMEM with 20\% FBS $(500 \mu \mathrm{l})$ was added to the lower chamber. Cells were collected following $24 \mathrm{~h}$ of treatment, as specified in the wound-healing assay method. Cells $\left(5 \times 10^{4}\right)$ in serum-free RPMI-1640 medium were added to the upper chamber and cultured for $24 \mathrm{~h}$ at $37^{\circ} \mathrm{C}$. The non-invading cells were removed by wiping gently with cotton swabs. The invading cells on the lower membrane were fixed with $100 \%$ methanol at room temperature for 15 min and stained with $0.1 \%$ crystal violet (Sigma-Aldrich; Merck $\mathrm{KGaA}$ ) at room temperature for $30 \mathrm{~min}$. The invading cells were detected by light microscopy (magnification, x100, BX53M; Olympus Corporation). The invasive capacity of cells was calculated as (number of invading cells in the treatment group/number of invading cells in the control group).

Tube formation assay. In the tube formation assay, HUVECs and ESCC KYSE70 cells were used to determine the rate of angiogenesis in a vasculogenic mimicry (VM) formation assay. Matrigel diluted with serum-free medium (1:1) was applied to a 96-well plate at $50 \mu \mathrm{l} /$ well, and incubated at $37^{\circ} \mathrm{C}$ for $1 \mathrm{~h}$. A total of $5 \times 10^{3}$ cells treated with drugs for $24 \mathrm{~h}$ suspended in $50 \mu \mathrm{l}$ culture medium were added to the matrix gel and incubated in $5 \% \mathrm{CO}_{2}$ at $37^{\circ} \mathrm{C}$ for $16 \mathrm{~h}$. The three-dimensional organization of the cells was examined and imaged under an inverted microscope (magnification, x100).

Western blotting. Total protein from EC9706 and KYSE70 cells treated for $24 \mathrm{~h}$ was extracted by lysis in solubilizing buffer with $1 \mathrm{mmol} / 1$ phenylmethanesulfonyl fluoride and a protease inhibitor cocktail (Beyotime Institute of Biotechnology, Haimen, China), and subsequently centrifuged at 13,000 x g at $4^{\circ} \mathrm{C}$ for $15 \mathrm{~min}$. Protein concentration was detected by a 
Bicinchoninic Acid protein measuring kit (Beyotime Institute of Biotechnology). A total of $30 \mu \mathrm{g}$ protein per lane was separated by $10 \%$ SDS-PAGE and transferred onto polyvinylidene fluoride membranes. The membranes were blocked with $5 \%$ skimmed milk at room temperature for $2 \mathrm{~h}$ and incubated with primary antibodies against cadherin-1 [Cell Signaling Technology (CST), Inc., Danvers, MA, USA; cat. no. 3195; dilution, 1:1,000], cadherin-2 (CST, Inc.; cat. no. 13116; dilution, 1:1,000), vimentin (CST, Inc.; cat. no. 5741; dilution, 1:1,000) and vascular endothelial growth factor (VEGF-A; Santa Cruz Biotechnology, Inc., Dallas, TX, USA; cat. no. sc-152; dilution, 1:500) overnight at $4^{\circ} \mathrm{C}$. $\beta$-actin (CST, Inc.; cat. no. 4970; dilution, 1:1,000) was utilized as a loading control. A horseradish peroxidase-conjugated goat anti-rabbit antibody (CST, Inc.; cat. no. 7074; dilution, 1:2,000) was subsequently applied at room temperature for $2 \mathrm{~h}$. An enhanced chemiluminescent detection reagent (Thermo Fisher Scientific, Inc.) was used to visualize the immunoreactive signals with a gel imaging system (Bio-Rad Laboratories, Inc.). Densitometry analysis of bands was calculated using Quantity One analysis software, version 4.62 (Bio-Rad Laboratories, Inc.).

Experimental tumor metastasis model. To evaluate the anti-metastatic effects of Aidi injection, nude mouse peritoneal metastasis models were established. A total of 24 female BALB/c NU mice (5 weeks old) were purchased from the Comparative Medicine Laboratory Animal Center (license no. scxk (SU) 2012-0004) of Yangzhou University (Jiangsu, China). These nude mice weighed $\sim 19 \mathrm{~g}$. The nude mice were housed at a constant temperature $\left(25-27^{\circ} \mathrm{C}\right)$, constant humidity (45-50\%), specific pathogen free environment. Sterile purified water and food were provided ad libitum. EC9706 cells $\left(2 \times 10^{6}\right)$ were inoculated into the peritoneal cavity of 20 mice, and the rest of 4 mice were not injected with the cells as the normal group. After 14 days following tumor implantation, the mice were treated with Aidi injection (1, 2 or $4 \mathrm{~g} / \mathrm{kg} /$ day), $60 \mathrm{mg} / \mathrm{kg}$ 5 -Fu, or the same volume of saline (control group) via intraperitoneal injection for 15 days. Each group contained four mice. At the end of treatment, the mice were sacrificed by cervical dislocation and a laparotomy was performed. The numbers of peritoneal cancer nodules were examined. All experimental procedures were performed following internationally accepted guidelines regarding the use of laboratory animals (18) and the protocol of the present study was reviewed and approved by the Institutional Animal Care and Use Committee of Yangzhou University (Jiangsu, China).

Immunohistochemistry. All tissues were fixed with $10 \%$ neutral buffered formalin at room temperature overnight and embedded in paraffin. Sections of 5- $\mu \mathrm{m}$ thickness of the tumor tissues temperature were cut for immunohistochemical staining. The slides were dewaxed in xylene and rehydrated a descending alcohol series. Hydrogen peroxide (3\%) was added to quench the endogenous peroxidase activity for $10 \mathrm{~min}$. Antigen retrieval was performed in citrate buffer at $95^{\circ} \mathrm{C}$ for 5 min. Goat serum (10\%; Beyotime Institute of Biotechnology) was used to block for $20 \mathrm{~min}$ at room temperature. Subsequently, the aforementioned primary antibodies (vimentin, dilution 1:200; cadherin-1, dilution 1:200; VEGF-A, dilution 1:100) were incubated with the sections at $4^{\circ} \mathrm{C}$ overnight. Following this incubation, the slides were incubated with an appropriate secondary antibody conjugated with HRP (CST, Inc.; undiluted; cat. no. 8114 ) at $37^{\circ} \mathrm{C}$ for $20 \mathrm{~min}$. The slides were stained with 3,3-diaminobenzidine at room temperature for $10 \mathrm{~min}$ following washing with PBS. The slides were counterstained with hematoxylin for $15 \mathrm{~min}$ at room temperature. The slides were subsequently dehydrated and mounted with neutral gum for imaging with inverted microscope (magnification, x200).

Statistical analysis. Each experiment was repeated three times. All values are presented as the mean \pm standard deviation. Data from the in vitro and in vivo experiments were compared using a one-way analysis of variance with Bonferroni's post hoc test, or a Student's t-test. $\mathrm{P}<0.05$ was considered to indicate a statistically significant difference. All analysis was performed using SPSS software version 16.0 (SPSS, Inc., Chicago, IL, USA).

\section{Results}

Aidi inhibits the viability of ESCC cells. EC9706 and KYSE70 cells treated with varying concentrations $(3,6,12,24,48$ or $96 \mathrm{mg} / \mathrm{ml}$ ) of Aidi for 24 or $48 \mathrm{~h}$ revealed significantly inhibited growth $(\mathrm{P}<0.05, \mathrm{P}<0.01 ;$ Fig. 1A) in a concentration and time-dependent manner. The cell growth rates of EC9706 for $24 \mathrm{~h}$ were $1.5 \mathrm{mg} / \mathrm{ml}(95.95 \pm 2.67 \%), 3 \mathrm{mg} / \mathrm{ml}(86.79 \pm 2.64 \%)$, $6 \mathrm{mg} / \mathrm{ml}(82.76 \pm 3.10 \%), 12 \mathrm{mg} / \mathrm{ml}(66.27 \pm 2.74 \%)$, $24 \mathrm{mg} / \mathrm{ml}(58.84 \pm 2.12 \%), 48 \mathrm{mg} / \mathrm{ml}(44.99 \pm 4.29 \%)$, $96 \mathrm{mg} / \mathrm{ml}(37.44 \pm 3.07 \%)$, respectively. The cell growth rates of KYSE70 for $24 \mathrm{~h}$ were $1.5 \mathrm{mg} / \mathrm{ml}(95.68 \pm 2.91 \%)$, $3 \mathrm{mg} / \mathrm{ml}(89.95 \pm 4.34 \%), 6 \mathrm{mg} / \mathrm{ml}(86.61 \pm 2.94 \%)$, $12 \mathrm{mg} / \mathrm{ml}(74.36 \pm 4.07 \%), 24 \mathrm{mg} / \mathrm{ml}(68.65 \pm 3.41 \%), 48 \mathrm{mg} / \mathrm{ml}$ $(50.11 \pm 3.24 \%), 96 \mathrm{mg} / \mathrm{ml}(39.57 \pm 3.87 \%)$, respectively. The half maximal inhibitory concentration at $24 \mathrm{~h}$ for EC9706 and KYSE70 cells was $25.89 \mathrm{mg} / \mathrm{ml}$ and $32.58 \mathrm{mg} / \mathrm{ml}$, respectively. Aidi was administered via intravenous injection once a day, so $24 \mathrm{~h}$ treatment was selected and Aidi concentrations of 6,12 and $24 \mathrm{mg} / \mathrm{ml}$ which growth inhibition rate was $<50 \%$ were used to avoid cytotoxic effects of excessive concentration in subsequent experiments; $32 \mathrm{mg} / \mathrm{ml} \mathrm{5-Fu}$ was used as a control. The morphological characteristics of the cells were observed following the selected treatments. The intercellular space increased, cell numbers were reduced and the cytoplasm shrank in response to Aidi and 5-Fu treatments compared with in the control group of the two cell lines (Fig. 1B).

Aidi suppresses the migration and invasion of ESCC cells. The migratory capacity of the cells was tested with a wound-healing assay. Treatment with Aidi for $24 \mathrm{~h}$ significantly inhibited the migration of EC9706 and KYSE70 cells in a dose-dependent manner(P<0.01; Fig.2A). The wound-healing rates of the EC9706 cells following treatment with 6,12 and $24 \mathrm{mg} / \mathrm{ml}$ Aidi and $32 \mathrm{mg} / \mathrm{ml} 5$-Fu were $84.77 \pm 3.46 \%, 76.88 \pm 2.27 \%, 46.70 \pm 1.65 \%$ and $30.44 \pm 3.85 \%$, respectively, while the wound-healing rates of the KYSE70 cells were $77.55 \pm 3.63 \%, 47.27 \pm 5.54 \%, 32.84 \pm 1.95 \%$ and $23.06 \pm 2.83 \%$, respectively. The trends of the rates in the two cell types, with different degrees of differentiation, are consistent. The number of invading cells was significantly reduced by treatment with Aidi, in a dose-dependent manner $(\mathrm{P}<0.01$; Fig. 2B). The invasion inhibition rates of treatment with 6,12 and $24 \mathrm{mg} / \mathrm{ml}$ Aidi and $32 \mathrm{mg} / \mathrm{ml} 5-\mathrm{Fu}$ were $76.46 \pm 1.45 \%$, 

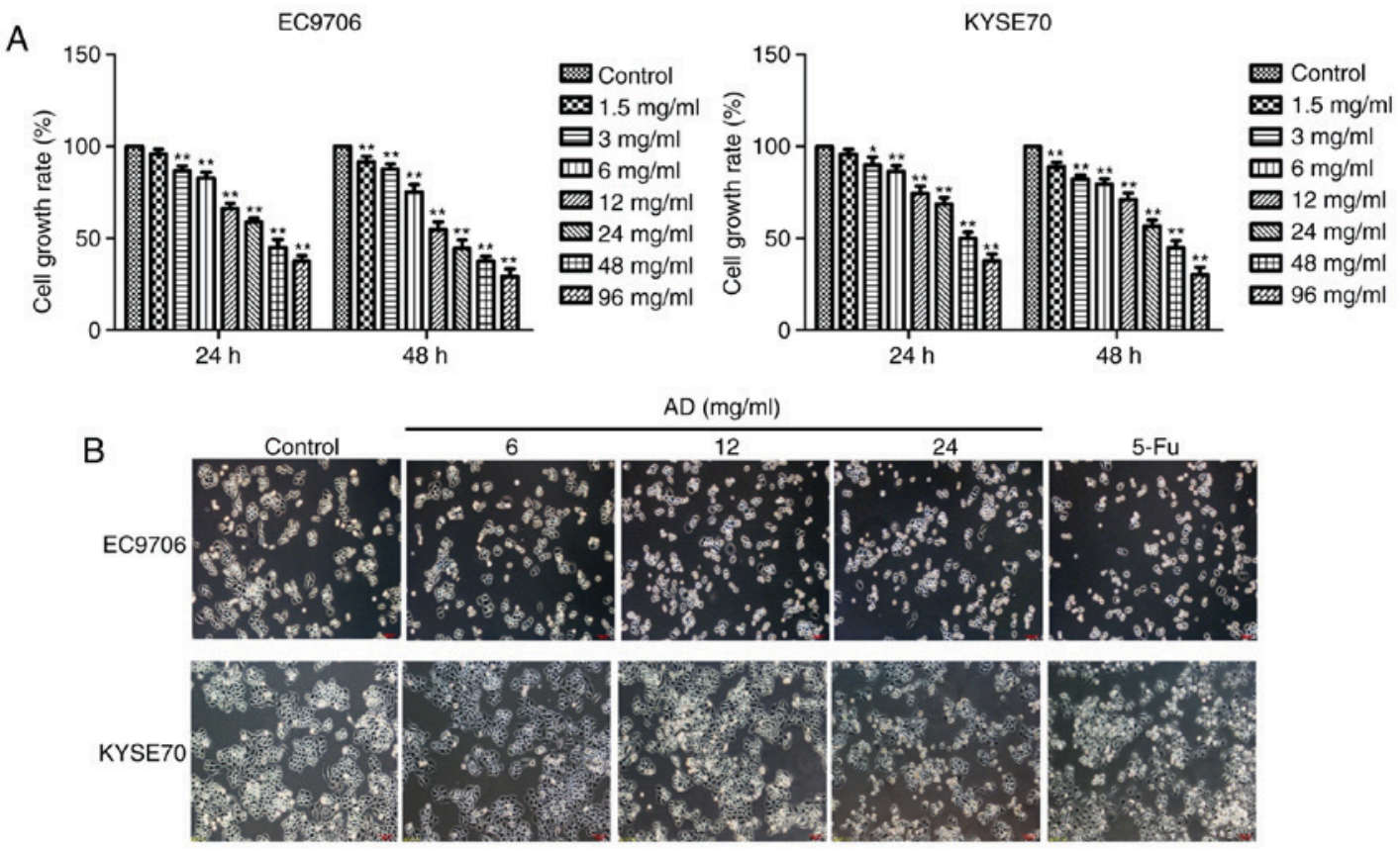

Figure 1. Effects of different concentrations of Aidi on cell growth in esophageal squamous cell carcinoma. (A) The cytotoxic effect of 24 or $48 \mathrm{~h}$ treatment with Aidi on EC9706 and KYSE70 cells. (B) The morphological characteristics of EC9706 and KYSE70 cells following treatment for 24 h. All values are presented as the mean \pm standard deviation. ${ }^{*} \mathrm{P}<0.05,{ }^{* *} \mathrm{P}<0.01$ vs. respective control. Magnification, $\mathrm{x} 100$. Scale bar, $100 \mu \mathrm{m}$. AD, Aidi; 5-Fu, 5-fluorouracil.
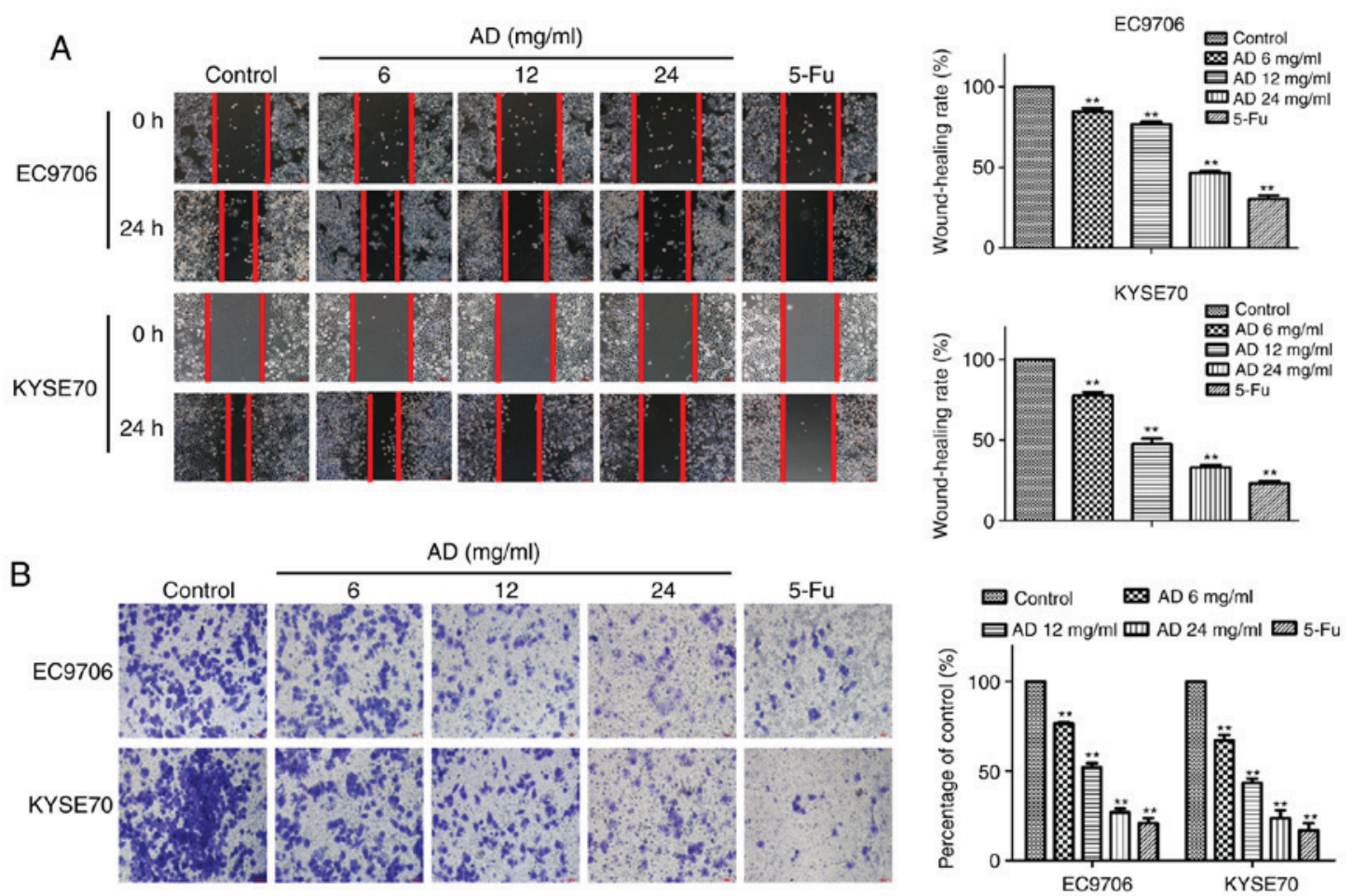

Figure 2. Effect of treatment with Aidi on the migration and invasion of esophageal squamous cell carcinoma cells. (A) The effect of Aidi on the migration of EC9706 and KYSE70 cells after $24 \mathrm{~h}$. (B) The effect of Aidi on the invasion of EC9706 and KYSE70 cells after $24 \mathrm{~h}$. All values are presented as the mean \pm standard deviation. ${ }^{* *} \mathrm{P}<0.01$ vs. respective control. Magnification, $\mathrm{x} 100$. Scale bar, $100 \mu \mathrm{m}$. AD, Aidi; 5-Fu, 5-fluorouracil.

$51.97 \pm 4.15 \%, 26.90 \pm 3.52 \%$ and $20.85 \pm 4.86 \%$ in EC9706 cells and $67.03 \pm 5.36 \%, 43.26 \pm 4.29 \%, 23.67 \pm 7.77 \%$ and $16.86 \pm 6.86 \%$ in KYSE70 cells, respectively. These results demonstrated that treatment with Aidi induced an inhibition of ESCC migratory and invasive capacity in a concentration-dependent manner, suggesting an inhibitory effect on metastasis.
Aidi inhibits the angiogenesis of ESCC. An angiogenesis assay was performed to investigate the effect of treatment with Aidi on angiogenesis in vitro. HUVECs resemble the principal constituent of tumor blood vessels; it was identified that treatment with Aidi may reduce the formation of a network of capillary-like tubes in HUVECs (Fig. 3A). To better 


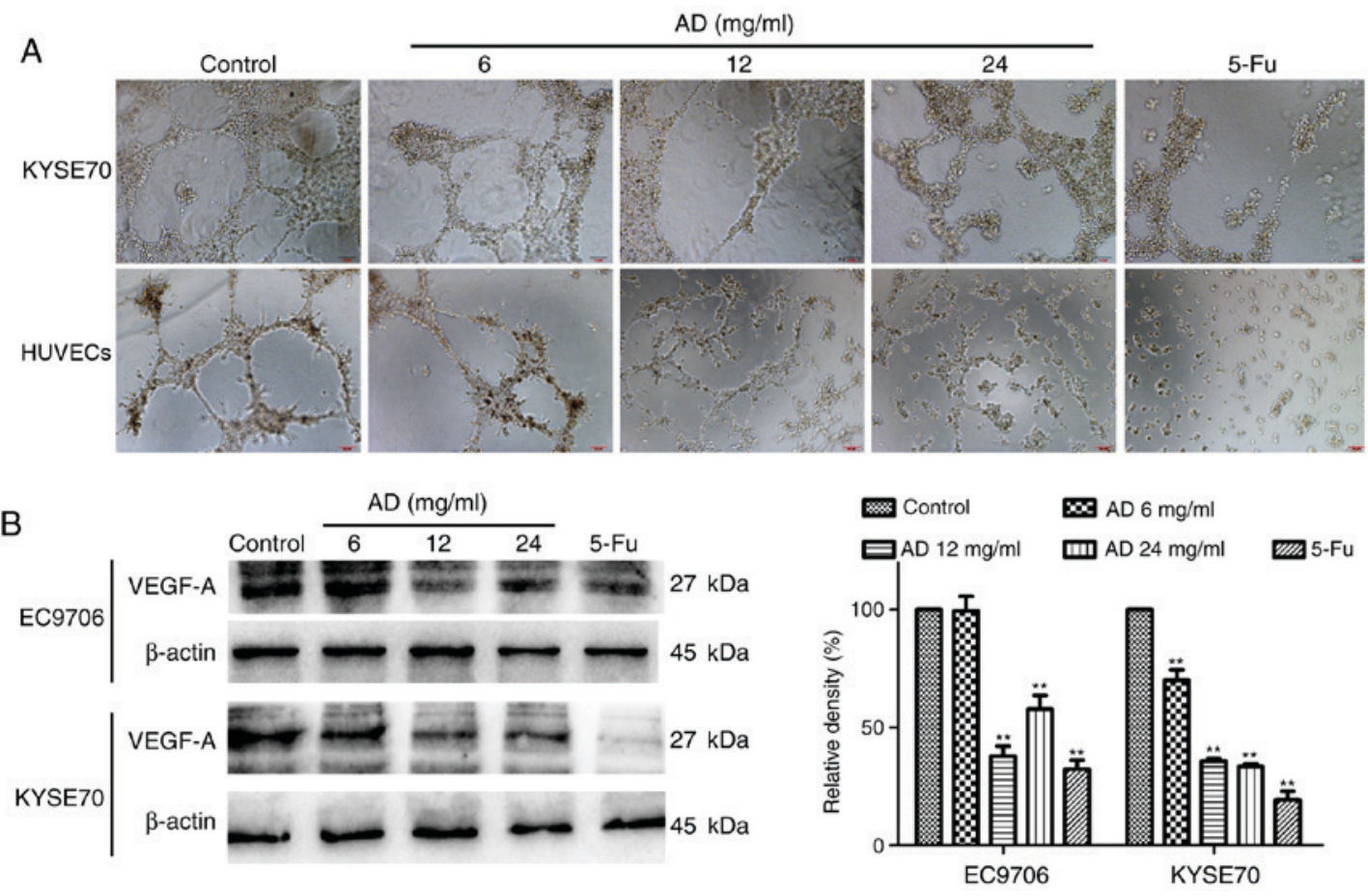

Figure 3. Effect of treatment with Aidi on angiogenesis and VM in HUVECs and esophageal squamous cell carcinoma cells. (A) The anti-angiogenesis effect of treatment with Aidi on HUVECs, and the anti-VM formation effect of treatment with Aidi on KYSE70 cells after 24 h. Magnification, x100. (B) Treatment with Aidi reduced the VEGF protein expression in EC9706 and KYSE70 cells. All values are presented as the mean \pm standard deviation. ${ }^{* *} \mathrm{P}<0.01$ vs. respective contro1. VM, vasculogenic mimicry; HUVECs, human umbilical vein endothelial cells; VEGF-A, vascular endothelial growth factor A; AD, Aidi; 5-Fu, 5-fluorouracil.

understand the mechanism of angiogenesis, VM formation in ESCC cells was examined. As EC9706 cells are highly differentiated and less malignant, they may not form capillary-like tubes well, while KYSE70 cells have higher malignancy, and were therefore selected. The results showed treatment with Aidi downregulated VM formation in KYSE70 cells (Fig. 3A). Western blot analysis demonstrated that treatment with Aidi decreased the expression of VEGF-A in EC9706 and KYSE70 cells (Fig. 3B). These data indicated that treatment with Aidi may inhibit angiogenesis.

Aidi inhibits epithelial-mesenchymal transition (EMT) signaling. EMT signaling contributes to tumor metastasis. To investigate whether the inhibition of EMT was associated with the effects of treatment with Aidi, EMT signaling in EC9706 and KYSE70 cells was examined. The expression levels of cadherin-1, associated with epithelial morphology, were increased, while the expression of cadherin-2 and vimentin, associated with mesenchymal morphology, were decreased in a dose-dependent manner following treatment with Aidi (Fig. 4).

Aidi injection inhibits peritoneal dissemination in an experimental metastasis model. To evaluate the effects of Aidi injection on the metastasis of ESCC, a mouse abdominal tumor model was established. The weight of nude mice in the treatment groups was compared with the negative control group, which were injected with saline; there was no significant alteration (Fig. 5A), indicating that no toxicity had occurred in any of the treated groups. Aidi injection inhibited peritoneal dissemination, as the number of macroscopic tumor nodules in the Aidi injection groups was fewer compared with that in the control group (Fig. 5B). These data suggested that Aidi injection may inhibit ESCC metastasis.

Aidi injection suppresses EMT and VEGF-A expression in peritoneal cancer nodules. To better understand the mechanism of Aidi injection on the suppression of metastasis in vivo, the expression levels of cadherin-1, vimentin and VEGF-A were examined in the tumor tissue of the nude mice by immunohistochemical analysis; cadherin-2 expression was not analyzed in the present study as the respective antibody could not be applied for immunohistochemical analysis. The results revealed that Aidi injection increased the expression of cadherin-1 and decreased the expression of vimentin and VEGF-A in the tumor tissue (Fig. 5C). The data collectively indicated that Aidi may suppress metastasis by inhibiting EMT and angiogenesis.

\section{Discussion}

Metastasis is the most fatal characteristic of cancer, accounting for $>90 \%$ of cancer-associated mortality (19). The process of cancer metastasis is complex, including alterations to the tumor microenvironment, cell growth and transformation, angiogenesis, invasion, dissemination, and the subsequent adhesion and colonization of a secondary organ or tissue (17). The identification of drugs that may prevent tumor metastasis is crucial. There is previous evidence to suggest that traditional Chinese medicine may serve a role in treating patients with cancer (20), including Aidi (21), shenmai (22), Brucea javanica (23) and Kanglaite ${ }^{\circledR}$ (24). Aidi is a Chinese 

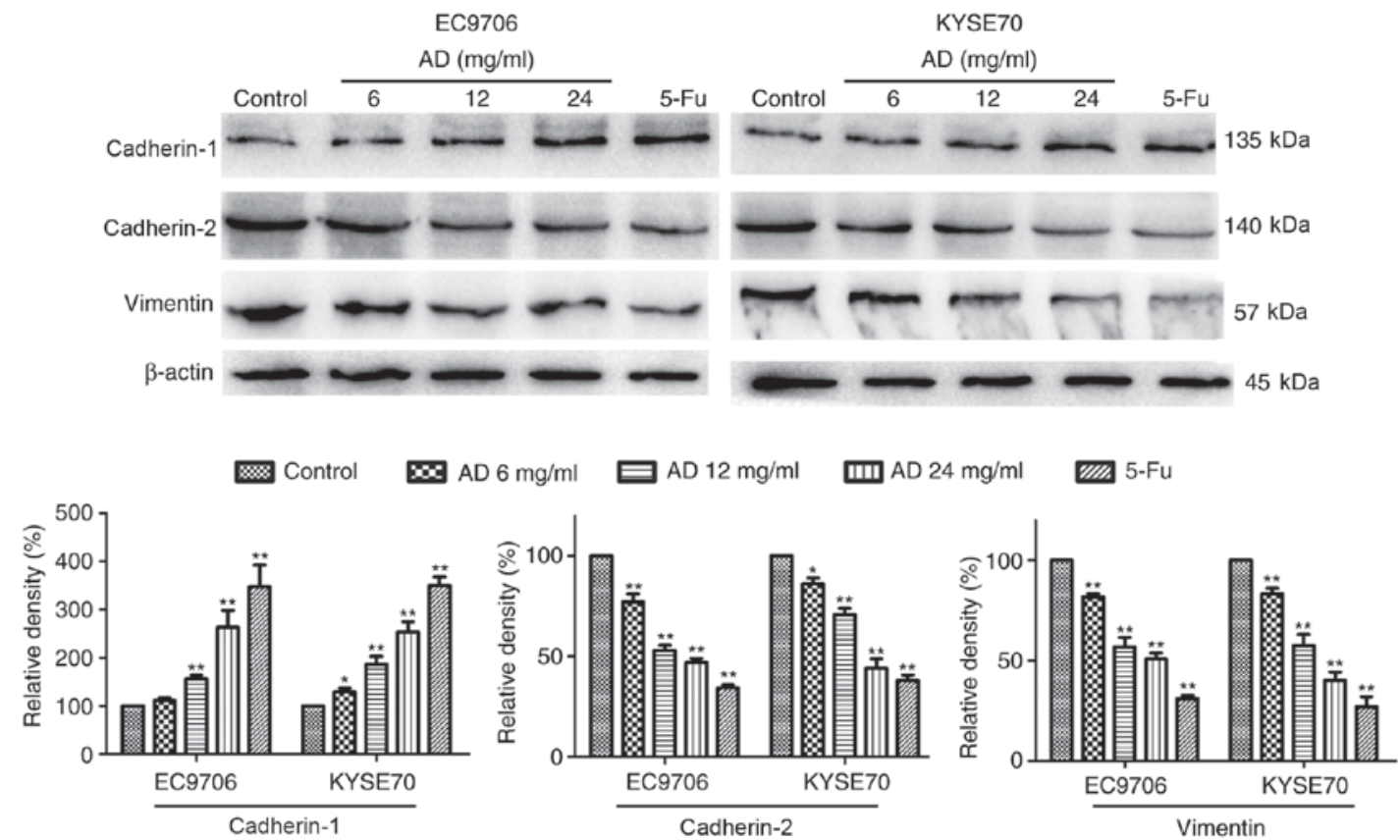

Figure 4. Effect of treatment with Aidi on epithelial-mesenchymal signaling in esophageal squamous cell carcinoma cells. Treatment with Aidi increased the level of cadherin-1, and decreased the expression of cadherin- 2 and vimentin, in a dose-dependent manner. All values are presented as the mean \pm standard deviation. ${ }^{*} \mathrm{P}<0.05,{ }^{* *} \mathrm{P}<0.01$ vs. respective control. AD, Aidi; 5-Fu, 5-fluorouracil.
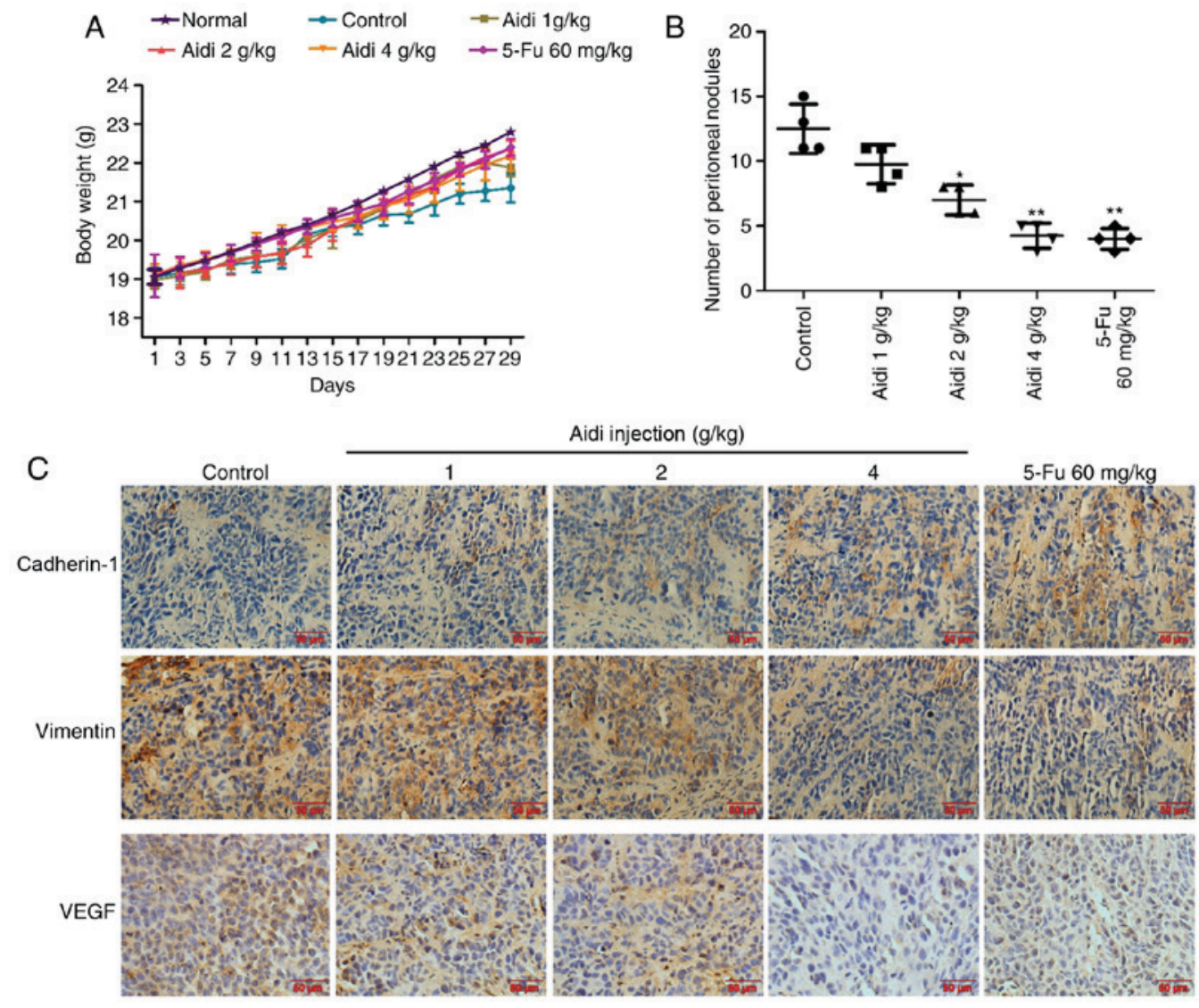

Figure 5. Effect of treatment with Aidi on tumor metastasis in vivo. (A) The effect of Aidi treatment on mouse body weight. (B) The number of peritoneal metastases in each group. All values are presented as the mean \pm standard deviation. ${ }^{*} \mathrm{P}<0.05,{ }^{* *} \mathrm{P}<0.01$ vs. control. (C) The expression of cadherin-1, vimentin and VEGF-A in the peritoneal dissemination nodules. Magnification, x200. Scale bar, $50 \mu \mathrm{m}$. VEGF-A, vascular endothelial growth factor-A; 5-Fu; 5-fluorouracil.

medicine extracted from Chinese drugs such as Mylabris, Radix ginseng, Astragalus membranaceus Bge and Radix
Acanthopanacis Senticosi (25). At present, Aidi injection is widely used in the treatment of esophageal cancer, lung cancer 
and colorectal carcinoma (10-14). However, whether Aidi may inhibit metastasis, and the underlying mechanisms of Aidi injection in ESCC, remain to be investigated. To identify whether Aidi injection is appropriate to prevent ESCC metastasis in clinical use, the effects of Aidi injection on viability, migration and invasion were observed. The present in vitro experimental results demonstrated that treatment with Aidi inhibited the viability, migration and invasion of EC9706 and KYSE70 cells in a dose-dependent manner. Furthermore, Aidi inhibited peritoneal dissemination in a mouse metastasis model. These present results demonstrated that Aidi injection may effectively reduce the extent of metastasis.

Angiogenesis is a principal driving force and an essential pathological feature of tumor progression, which serves a key role in tumor metastasis and metabolic deregulation (26). Tumor angiogenesis is a popular research topic in the clinical treatment of cancer (27). There are two characteristic types of angiogenesis in tumors; blood vessels formed by normal endothelial cells and VM, vessel-like structures lined with tumor cells instead of endothelial cells (28). The present data demonstrated that treatment with Aidi may inhibit the tube formation of HUVECs and the VM formation of KYSE70 cells. VEGF-A is a key growth factor for endothelial cells in tumor angiogenesis (29); higher VEGF-A expression is associated with a more advanced Tumor, Node, Metastasis stage and a decreased overall survival in patients with esophageal cancer (30). In the present study, Aidi treatment decreased the expression of VEGF-A in HUVECs and KYSE70 cells. VEGF-A expression in the mouse tumor tissue, as determined by immunohistochemistry, revealed the same trend.

EMT is an important process in tumor progression, in which epithelial cells undergo a phenotypic conversion to mesenchymal cells, which is closely associated with invasion, metastasis and prognosis (31). A reduction in cadherin-1 expression, and an increase in cadherin-2 and vimentin expression, are representative characteristics of EMT (32). The present study demonstrated that treatment with Aidi in EC9706 and KYSE70 cells increased the levels of cadherin-1, and decreased the expression of cadherin-2 and vimentin, in a dose-dependent manner, representing the inhibition of EMT signaling. In vivo, Aidi injection additionally increased the expression of cadherin-1 and decreased the expression of vimentin in an implanted tumor model of human ESCC in nude mice.

Additionally, EMT may promote angiogenesis and VM formation. Recent studies have demonstrated that transcription factors associated with EMT, including Twist-related protein 1 (TWIST1) and zinc-finger E-box-binding homeobox 1 (ZEB1), serve a key role in angiogenesis in cancer $(33,34)$. Sun et al (35) identified that the low expression of TWIST1 in liver cancer cells was associated with a decreased capacity for VM formation. Liu et al (34) observed that ZEB1 served an important role in the VM process. A future direction of research may be to verify whether treatment with Aidi inhibits tumor angiogenesis through an effect on EMT and to further clarify the mechanism of the anti-metastatic effect of treatment with Aidi on ESCC.

Aidi, a medicine extracted from Chinese drugs, exhibits anti-tumor activity. The present data indicated that treatment with Aidi may inhibit ESCC cell proliferation, migration, invasion, angiogenesis and EMT. There was evidence that treatment with Aidi effectively suppressed tumor metastasis in a nude mouse model through the reduced expression of vimentin and VEGF-A, and the increased expression of cadherin-1 in the tumor tissue. Aidi may inhibit tumor metastasis by inhibiting EMT and angiogenesis in human ESCC. The present results highlighted the anti-metastatic activity of Aidi, potentially providing a theoretical basis for its clinical use.

\section{Acknowledgements}

We thank Dr Yang Bao (The Affiliated Hospital of Yangzhou University, Yangzhou, China) the support in statistical analysis.

\section{Funding}

No funding was received.

\section{Availability of data and materials}

The datasets used and/or analyzed during the current study are available from the corresponding author on reasonable request.

\section{Authors' contributions}

QS and ZD made substantial contributions to the design of the present study. QS, YD and FJ conducted the experiments. QS and YD made substantial contributions of data analysis. ZD supervised all the work and critically revised the manuscript for important intellectual content. All the authors have approved the final manuscript.

\section{Ethics approval and consent to participate}

All experimental procedures were performed following internationally accepted guidelines regarding the use of laboratory animals, and the protocol of the present study was reviewed and approved by the Institutional Animal Care and Use Committee of Yangzhou University (Jiangsu, China).

\section{Consent for publication}

Not applicable.

\section{Competing interests}

The authors declare that they have no competing interests.

\section{References}

1. Chen W, Zheng R, Baade PD, Zhang S, Zeng H, Bray F, Jemal A, Yu XQ and He J: Cancer statistics in China, 2015. CA Cancer J Clin 66: 115-132, 2016.

2. Li S, Qin X, Chai S, Qu C, Wang X and Zhang H: Modulation of E-cadherin expression promotes migration ability of esophageal cancer cells. Sci Rep 6: 21713, 2016.

3. Zhang L, Ma J, Han Y, Liu J, Zhou W, Hong L and Fan D: Targeted therapy in esophageal cancer. Expert Rev Gastroenterol Hepatol 10: 595-604, 2016.

4. Domper Arnal MJ, Ferrández Arenas Á and Lanas Arbeloa Á: Esophageal cancer: Risk factors, screening and endoscopic treatment in Western and Eastern countries. World J Gastroenterol 21: 7933-7943, 2015. 
5. Zeng H, Zheng R, Zhang S, Zuo T, Xia C, Zou X and Chen W: Esophageal cancer statistics in China, 2011: Estimates based on 177 cancer registries. Thorac Cancer 7: 232-237, 2016.

6. Jain R, Gupta S, Pasricha N, Faujdar M, Sharma M and Mishra P: ESCC with metastasis in the young age of caustic ingestion of shortest duration. J Gastrointest Cancer 41: 93-95, 2010.

7. Ludmir EB, Robey B, Shelby E, Patel-Nguyen SV, Rittershaus A and Contarino MR: Skeletal muscle metastasis from signet ring cell esophageal adenocarcinoma. Transl Gastroenterol Hepatol 1: 37, 2016.

8. Wang H, Deng F, Liu Q and Ma Y: Prognostic significance of lymph node metastasis in esophageal squamous cell carcinoma. Pathol Res Pract 213: 842-847, 2017.

9. Xiao Z, Wang C, Zhou R, Hu S, Yi N, Feng J, Zhou M, Liu S, Chen L, Ding J, et al: Can Aidi injection improve overall survival in patients with non-small cell lung cancer? A systematic review and meta-analysis of 25 randomized controlled trials. Complement Ther Med 37: 50-60, 2018.

10. Xu J, Ju WZ and Tan HS: Study of pharmacological effects and clinical application of Aidi injection. Pharm Clin Res 1: 48-51, 2012 (In Chinese).

11. Zhang $\mathrm{H}$, Jiang $\mathrm{H}, \mathrm{Hu} \mathrm{X}$ and Jia Z: Aidi injection combined with radiation in the treatment of non-small cell lung cancer: A meta-analysis evaluation the efficacy and side effects. Cancer Res Ther 11 (Suppl 1): C118-C121, 2015.

12. Lou HZ, Pan HM and Jin W: Clinical study on treatment of primary liver cancer by Aidi injection combined with cool-tip radiofrequency ablation. Zhongguo Zhong Xi Yi Jie He Za Zhi 27: 393-395, 2007 (In Chinese).

13. Ji B and Yuan J: Meta-analysis of the clinical efficacy and safety about Aidi injection in the treatment of colorectal cancer. China Pharm 40: 3797-3799, 2011 (In Chinese).

14. Zhang MM, Liu YL, Chen Z, Li XR, Xu QM and Yang SL: Studies on chemical constituents from Aidi injection. Chin Tradit Herbal Drugs 8: 1462-1470, 2012 (In Chinese).

15. Jiancheng W, Long G, Ye Z, Jinlong L, Pan Z, Lei M and Kehu Y: Effect of aidi injection plus chemotherapy on gastric carcinoma: A meta-analysis of randomized controlled trials. J Tradit Chin Med 35: 361-74, 2015

16. Wang Q, He X, Tian J, Wang X, Ru P, Ruan Z and Yang K: A meta-analysis of aidi injection plus taxotere and cisplatin in the treatment of non-small cell lung cancer. Zhongguo Fei Ai Za Zhi 13: 1027-1034, 2010 (In Chinese).

17. Jin $X, Z$ Zhu Z and Shi Y: Metastasis mechanism and gene/protein expression in gastric cancer with distant organs metastasis. Bull Cancer 101: E1-E12, 2014.

18. Jones-Bolin S: Guidelines for the care and use of laboratory animals in biomedical research. Curr Protoc Pharmacol Appendix 4: Appendix 4B, 2012. doi: 10.1002/0471141755.pha04bs59.

19. Robert J: Biology of cancer metastasis. Bull Cancer 100: 333-342, 2013 (In French).

20. Tao W, Luo X, Cui B, Liang D, Wang C, Duan Y, Li X, Zhou S, Zhao M, Li Y, et al: Practice of traditional Chinese medicine for psycho-behavioral intervention improves quality of life in cancer patients: A systematic review and meta-analysis. Oncotarget 6: 39725-39739, 2015.

21. Xiao Z, Liang R, Wang CQ, Xu S, Li N, He Y, Tang F, Chen L and $\mathrm{Ma} \mathrm{H}$ : Can aidi injection alleviate the toxicity and improve the clinical efficacy of radiotherapy in lung cancer? A meta-analysis of 16 randomized controlled trials following the PRISMA guidelines. Medicine (Baltimore) 95: e4517, 2016.

22. Wang L, Huang XE and Cao J: Clinical study on safety of cantharidin sodium and shenmai injection combined with chemotherapy in treating patients with breast cancer postoperatively. Asian Pac J Cancer Prev 15: 5597-600, 2014.
23. Ji ZQ, Huang XE, Wu XY, Liu J, Wang L and Tang JH: Safety of Brucea javanica and cantharidin combined with chemotherapy for treatment of NSCLC patients. Asian Pac J Cancer Prev 15: 8603-8605, 2014

24. Liu Y, Zhang W, Wang XJ and Liu S: Antitumor effect of Kanglaite ${ }^{\circledR}$ injection in human pancreatic cancer xenografts. BMC Complement Altern Med 14: 228, 2014

25. Xiao Z, Wang C, Chen L, Tang X, Li L, Li N, Li J, Gong Q, Tang F, Feng $\mathbf{J}$ and Li X: Has aidi injection the attenuation and synergistic efficacy to gemcitabine and cisplatin in non-small cell lung cancer? A meta-analysis of 36 randomized controlled trials. Oncotarget 8: 1329-1342, 2017.

26. Wang Z, Dabrosin C, Yin X, Fuster MM, Arreola A, Rathmell WK, Generali D, Nagaraju GP, El-Rayes B, Ribatti D, et al: Broad targeting of angiogenesis for cancer prevention and therapy. Semin Cancer Biol 35 (Suppl): S224-S243, 2015.

27. Yu J, Zhang Y, Leung LH, Liu L, Yang F and Yao X: Efficacy and safety of angiogenesis inhibitors in advanced gastric cancer: a systematic review and meta-analysis. J Hematol Oncol 9: 111, 2016.

28. Qiao L, Liang N, Zhang J, Xie J, Liu F, Xu D, Yu X and Tian Y: Advanced research on vasculogenic mimicry in cancer. J Cell Mol Med 19: 315-326, 2015.

29. Gianni-Barrera R, Trani M, Fontanellaz C, Heberer M, Djonov V, Hlushchuk R and Banfi A: VEGF over-expression in skeletal muscle induces angiogenesis by intussusception rather than sprouting. Angiogenesis 16: 123-136, 2013.

30. Xu XL, Ling ZQ, Chen W, Xu YP and Mao WM: The overexpression of VEGF in esophageal cancer is associated with a more advanced TMN stage: A meta-analysis. Cancer Biomark 13: 105-113, 2013.

31. Davis FM, Stewart TA, Thompson EW and Monteith GR: Targeting EMT in cancer: Opportunities for pharmacological intervention. Trends Pharmacol Sci 35: 479-488, 2014.

32. Banyard $\mathbf{J}$ and Bielenberg DR: The role of EMT and MET in cancer dissemination. Connect Tissue Res 56: 403-413, 2015.

33. Che N, Zhao XL, Sun T, Zhao XM, Gu Q, Dong XY, Zhao N, Liu YR, Yao Z and Sun BC: The role of Twist1 in hepatocellular carcinoma angiogenesis: A clinical study. Hum Pathol 42: 840-847, 2011

34. Liu Z, Sun B, Qi L, Li H, Gao J and Leng X: Zinc finger E-box binding homeobox 1 promotes vasculogenic mimicry in colorectal cancer through induction of epithelial-to-mesenchymal transition. Cancer Sci 103: 813-820, 2012.

35. Sun T, Zhao N, Zhao XL, Gu Q, Zhang SW, Che N, Wang XH, Du J, Liu YX and Sun BC: Expression and functional significance of Twist1 in hepatocellular carcinoma: Its role in vasculogenic mimicry. Hepatology 51: 545-556, 2010.

This work is licensed under a Creative Commons Attribution-NonCommercial-NoDerivatives 4.0 International (CC BY-NC-ND 4.0) License. 